\section{A visão masculina sobre métodos contraceptivos em uma comunidade rural da Bahia, Brasil}

\author{
Male views of contraceptive methods \\ in a rural community in Bahia State, Brazil
}

\begin{abstract}
Family planning programs have traditionally concentrated on women. This study aimed to determine men's knowledge of contraceptive methods in a rural community in Bahia State, Brazil. Mean age of interviewees was 40.0 ( \pm 17.6$)$ years. Avoiding unwanted pregnancy was reported as a responsibility of the couple by $39.7 \%$ of the male interviewees $(n=71)$ and as the man's responsibility by $26.8 \%(n=48)$. The most widely known methods were condoms (98.9\%) and the pill (96.6\%). Condoms (22.9\%), female sterilization (21.2\%), and the pill (12.8\%) were the most widely used methods. The majority of the interviewees (56.4\%) reported that they "always" use some method. The men chose the method in $45.6 \%$ of the couples. The results indicate that more options for contraceptive methods should be offered, thereby facilitating the best choice of methods by the couple. Still,since in this study men chose the method in nearly half of the cases, it is necessary to prioritize couples' participation in family planning programs.
\end{abstract}

Contraception; Rural Communities; Gender
Danilo Cerqueira do Espírito-Santo 1 José Tavares-Neto 2

\section{Introdução}

Dentre os tópicos da Saúde Reprodutiva, a questão do planejamento familiar é uma das mais debatidas; ainda porque, por possuir implicações sócio-demográficas e econômicas, esta é uma atividade que não diz respeito somente ao casal que o pratica, mas sim a toda a sociedade, sendo uma das mais importantes ações preventivas na área da saúde, ao proporcionar aos casais as informações e os meios necessários na decisão de ter uma prole de forma consciente e voluntária. O planejamento familiar é de livre decisão do casal 1, cabendo ao Estado a tarefa de propiciar recursos educacionais e científicos ao exercício desse direito.

Discussões sobre a Saúde Reprodutiva, há algum tempo, vêm sofrendo um processo de legitimação, principalmente após a Conferência Internacional de População e Desenvolvimento (CIPD), no Cairo, em 1994; da Cúpula Mundial de Desenvolvimento Social (CMDS), em Copenhague, 1995; e da IV Conferência Mundial sobre a Mulher (CMM), em Pequim, 1995; contudo, a absorção do significado destas por parte dos organismos políticos é heterogênea 2 .

Ainda, desde 1983, por meio de uma proposta inovadora (à época), no âmbito do Programa de Assistência Integral à Saúde da Mulher (PAISM), e baseada no conceito de Atenção Integral à Saúde da Mulher (AISM), os Programas de Planejamento Familiar passaram a 
privilegiar a mulher no atendimento das questões vinculadas à reprodução 3. Dessa forma, tais programas, salvo algumas iniciativas pontuais de inclusão dos homens 4, vinham desde então se preocupando quase que exclusivamente em atingir a população feminina. Isso, de certo, veio contribuir substancialmente para que a grande maioria da clientela que participava das atividades do planejamento familiar fosse, durante a década de 90, do sexo feminino 5 . Por outro lado, a regulação da fecundidade é de interesse das mulheres, quer seja pelo fato de, tradicionalmente, conhecerem as conseqüências e os eventuais riscos da gestação, quer seja porque assumem as maiores responsabilidades pelo cuidado dos filhos. Isso também, provavelmente, explica a maior demanda feminina pelos Programas de Planejamento Familiar 5 .

Segundo Duarte 6, a grande maioria das pessoas do sexo masculino conhece diferentes métodos contraceptivos. Não obstante, é provável que a depender das características socioculturais da comunidade, o nível de conhecimento sobre esses métodos seja variável. Como observado por Carvalho et al. 7, as pessoas do sexo masculino participam decisivamente do processo de regulação da fecundidade do casal, mesmo quando o método utilizado é feminino. É sabido, também, que a influência exercida pelos homens sobre as respectivas companheiras, ainda é notória na sociedade brasileira, principalmente em comunidades pobres do interior do país, onde as mulheres continuam dependentes, cultural e economicamente, de seus cônjuges ou companheiros; apesar de, atualmente, se observar uma tendência a maiores conquistas por conta da população feminina e uma redefinição do papel do homem e do masculino 8 .

Desse modo, ao valorizar o conhecimento dos homens sobre os métodos contraceptivos, o programa de planejamento familiar existente, ou a ser implementado, terá mais informações relevantes para o melhor planejamento das formas de atuação. Porque deixa de ter como prioridade a atenção à mulher e passa a programar também atividades que visem ao casal com todas as suas peculiaridades. Dessa forma, os Programas de Planejamento Familiar poderiam atingir com mais eficácia os seus objetivos, visto que levariam em conta as características culturais dessas comunidades e das pessoas envolvidas. Com estes pressupostos, esta investigação buscou analisar qual é o grau de conhecimento sobre diferentes métodos contraceptivos e a freqüência de utilização dos mesmos, numa população masculina de comunidade carente e rural da região do semiárido do Estado da Bahia.

\section{Material e métodos}

A população escolhida para o estudo foi a do povoado de Cavunge, no Município de Ipecaetá, que dista $168 \mathrm{~km}$ de Salvador e $57 \mathrm{~km}$ de Feira de Santana, no Estado da Bahia. A área do povoado de Cavunge é de aproximadamente $63,5 \mathrm{~km}^{2}$ e no ano de 2000 tinha uma população de 2.049 habitantes, sendo 911 (44,5\%) residentes na sede do povoado e $1.138(55,5 \%)$ na área rural; no total, aproximadamente um terço $(29,0 \%)$ ou 595 pessoas eram do sexo masculino com 18 ou mais anos de idade 9 .

Este estudo de corte transversal utilizou uma amostra de conveniência, selecionada por sorteio com base no registro do morador no banco de dados de um censo realizado previamente no povoado ${ }^{9}$. Após isso, com a seleção aleatória de duzentos homens maiores de 17 anos de idade, os dados foram coletados por meio de entrevista individual, realizada por um dos autores do projeto, nas residências dos selecionados e utilizando um questionário com perguntas objetivas e pré-codificadas. Para avaliar o conhecimento, foi perguntado se o entrevistado sabia o que era cada um dos métodos contraceptivos citados nos resultados. Caso tivessem respondido corretamente a pergunta anterior, os entrevistados foram solicitados a explicar como o método evitava que a mulher engravidasse; se a explicação estivesse correta segundo critérios do Manual para Prestação de Serviços em Saúde Reprodutiva 10, o entrevistado seria considerado conhecedor do mecanismo de ação do método. Quando, após três tentativas, foi impossível entrevistar a pessoa selecionada, a mesma foi excluída da pesquisa.

Os critérios de inclusão no estudo foram: ser morador com residência fixa no povoado de Cavunge, com idade superior a 17 anos e concordância em participar do estudo, mediante assinatura do Termo de Consentimento Livre e Esclarecido, em conformidade com a Resolução CONEP no 196 de 199611 e parecer do Comitê de Ética em Pesquisa do Centro de Pesquisas Gonçalo Moniz, Fundação Oswaldo Cruz.

Na análise estatística foi utilizado o programa SPSS for Windows versão 9.0. As variáveis 
quantitativas foram analisadas pelo teste $\mathrm{t}$ de Student ou não-paramétrico (teste de MannWhitney), conforme indicação. Para testar a correlação entre variáveis utilizou-se o coeficiente de correlação de Spearman, pelo fato das variáveis não terem distribuição normal. Os resultados das análises estatísticas foram considerados significantes quando a probabilidade de erro tipo I foi $\leq 5 \%(p \leq 0,05)$.

A execução deste estudo transcorreu de forma a cumprir os princípios éticos da Declaração de Helsinki.

\section{Resultados}

\section{Características sócio-demográficas}

O número amostral foi de 179 pessoas, o que correspondeu a $30,1 \%$ dos homens maiores de 17 anos de idade $(n=595)$, moradores em Cavunge no ano de 2000. Entre as características sócio-demográficas da população estudada (Tabela 1), a média de idade dos entrevistados foi de 40,0 $( \pm 17,6)$ anos, mediana de 36 e limites de 18 e 86 anos. A maioria ( $n=167 ; 93,3 \%$ ) residia no povoado pelo tempo médio de 31 anos e 3 meses, sendo positiva a correlação entre a idade e o tempo de residência no povoado $(r=0,64$; $\mathrm{p}=10^{-6}$ ). A fonte de renda principal se concentra em atividades que não necessitam de qualificação formal: lavoura, biscate, dentre outras, em $84,4 \%(n=151)$ dos casos e onze $(6,1 \%)$ pessoas viviam exclusivamente da aposentadoria. Setenta $(39,1 \%)$ eram analfabetos e entre estes a média de idade foi de $52,6( \pm 17,2)$ anos, apenas sete $(3,9 \%)$ pessoas estavam cursando ou haviam concluído o ensino médio, com a média de idade de $22,4( \pm 6,8)$ anos. A diferença entre as médias de idade dos analfabetos e daqueles que estão cursando ou já cursaram o ensino médio foi estatisticamente diferente $(\mathrm{p}<$ $0,001)$. A maioria $(74,9 \%)$ dos entrevistados $(\mathrm{n}=$ 134) tinha relacionamento sexual fixo (heterossexual, com práticas sexuais com a mesma companheira por nove ou mais meses). O número médio de filhos foi de $4,1( \pm 3,3)$ filhos.

\section{Responsabilidade sobre questões conjugais}

A responsabilidade de educar e cuidar dos filhos (Tabela 2) foi referida como responsabilidade do casal por $112(62,6 \%)$ dos participantes; cinqüenta e dois $(29,1 \%)$ acharam que esta tarefa era exclusivamente feminina e treze $(7,3 \%)$ consideraram como uma atividade paterna. Quanto à preocupação com a prevenção de gravidez indesejada, foi referida como res-
Características sócio-demográficas e a freqüência de relacionamento sexual fixo na amostra populacional estudada ( $n=179)$. Cavunge, Ipecaetá, Bahia, Brasil.

\begin{tabular}{|c|c|c|}
\hline & $\mathbf{n}$ & $\%$ \\
\hline \multicolumn{3}{|l|}{ Idade } \\
\hline $18-22$ & 36 & 20,1 \\
\hline $23-33$ & 38 & 21,2 \\
\hline $34-41$ & 34 & 19,0 \\
\hline $42-56$ & 36 & 20,1 \\
\hline $57-86$ & 35 & 19,6 \\
\hline \multicolumn{3}{|l|}{ Naturalidade } \\
\hline Ipecaetá ou região & 167 & 93,3 \\
\hline Outra cidades & 12 & 6,7 \\
\hline \multicolumn{3}{|l|}{ Ocupação principal } \\
\hline Sem qualificação formal & 151 & 84,4 \\
\hline Com qualificação formal & 3 & 1,7 \\
\hline Aposentado & 11 & 6,1 \\
\hline Estudante & 14 & 7,8 \\
\hline \multicolumn{3}{|l|}{ Escolaridade } \\
\hline Analfabeto & 70 & 39,1 \\
\hline 1으 grau incompleto & 92 & 51,4 \\
\hline 1ㅇ grau completo & 10 & 5,6 \\
\hline 2o grau incompleto & 3 & 1,7 \\
\hline 2o grau completo & 4 & 2,2 \\
\hline \multicolumn{3}{|l|}{ Relacionamento sexual fixo* } \\
\hline Sim & 131 & 73,2 \\
\hline Não & 48 & 26,8 \\
\hline
\end{tabular}

* Relacionamento heterossexual e práticas sexuais com a mesma companheira, por no mínimo nove meses.

ponsabilidade do casal por $71(39,7 \%)$, da mulher por 51 (28,5\%) e do homem por $48(26,8 \%)$ dos entrevistados.

\section{Conhecimento sobre métodos contraceptivos}

A Tabela 3 mostra que o conhecimento de pelo menos um método contraceptivo foi elevado $(97,2 \% ; n=174)$, sendo as principais fontes de informação sobre os métodos os meios de comunicação de massa, referidos como única fonte por $24(13,8 \%)$ dos entrevistados. Oitenta e sete $(50,0 \%)$ consideraram duas ou mais fontes de informação. O método contraceptivo mais freqüentemente referido (Tabela 4) foi a "camisinha” por $98,9 \%(n=177)$ das pessoas, seguido 
da pílula anticoncepcional $(96,6 \% ; \mathrm{n}=173) \mathrm{e}$ da laqueadura tubária $(89,9 \%$; $n=161)$. Os métodos menos conhecidos foram o diafragma $(22,9 \%)$, o creme vaginal $(35,8 \%)$ e o DIU $(46,4 \%)$. Houve também grande porcentagem de conhecimento sobre os anticoncepcionais injetáveis $(82,7 \%)$, mas somente o conhecimento sobre o coito interrompido $(\mathrm{p}<0,04)$ e a vasectomia ( $p<0,008$ ) foram mais freqüentes, respectivamente, nos menores de 37 anos e entre aqueles com 37 ou mais anos de idade.

\section{Conhecimento dos mecanismos de ação e uso dos métodos contraceptivos}

O conhecimento sobre os mecanismos de ação dos métodos contraceptivos foi pequeno (Tabela 5). Somente os mecanismos da "camisinha” $(64,2 \%)$ e do coito interrompido $(60,9 \%)$ são conhecidos pela maioria dos entrevistados. O conhecimento sobre os mecanismos de ação dos demais métodos foi inferior a 4,0\%.

Apesar da maioria $(56,4 \% ; n=101)$ ter referido "sempre" utilizar algum método contraceptivo, esta é uma porcentagem muito pequena, não diferindo $(\mathrm{p}>0,16)$ entre aqueles com e aqueles sem relacionamento fixo $(59,5 \% ; n=78$ e $47,9 \% ; n=23$, respectivamente), mas significativamente maior $(\mathrm{p}<0,02)$ entre aqueles com idade menor ou igual à mediana do que entre aqueles com idade superior à mediana $(65,6 \%$; $\mathrm{n}=59$ e $41,6 \% ; \mathrm{n}=42$, respectivamente). O mais utilizado é a "camisinha”, referido como utilizado em todas as relações por $22,9 \%(n=41)$ dos entrevistados, seguido pela laqueadura $21,2 \%(n=38)$ e pela pílula com $12,8 \%(n=23)$. Os demais métodos atingiram uma proporção de uso inferior a $4,0 \%$, sendo que a vasectomia, o DIU, o creme vaginal e o diafragma foram referidos como utilizados por apenas um ou nenhum dos casais.

\section{Opção pelo método}

Dentre aqueles que utilizavam algum método contraceptivo $(63,7 \% ; n=114)$, a opção pelo método era realizada em quase metade dos casos pelo homem $(45,6 \% ; n=52)$ dos casais; já a mulher foi descrita como a responsável por $24,6 \%$ dos participantes $(n=28)$. Nos demais casos, o médico ou profissional de saúde em $14,0 \%(n=16)$, o casal em $11,4 \%(n=13)$, entre outros $(4,4 \% ; n=5)$, eram os responsáveis pela escolha do método. Sessenta e cinco entrevistados $(36,3 \%)$ revelaram não utilizar nenhum método contraceptivo.

Apenas três entrevistados $(1,7 \%)$ não haviam mantido relação sexual e quatro $(2,2 \%)$
Tabela 2

Responsabilidade referida sobre o cuidado dos filhos e a prevenção da gravidez não-planejada, pelas 179 pessoas do sexo masculino, maiores de 17 anos. Cavunge, Ipecaetá, Bahia, Brasil.

\begin{tabular}{lrr}
\hline & $\mathbf{n}$ & \multicolumn{1}{c}{$\%$} \\
\hline $\begin{array}{lrr}\text { Cuidado dos filhos } \\
\text { Homem }\end{array}$ & 13 & 7,3 \\
Mulher & 52 & 29,1 \\
Ambos & 112 & 62,6 \\
Não quis responder & 2 & 1,1 \\
& & \\
Prevenção da gravidez & & \\
não-planejada & & \\
Homem & 48 & 26,8 \\
Mulher & 51 & 28,5 \\
Ambos & 71 & 39,7 \\
Não quis responder & 9 & 5,0 \\
\hline
\end{tabular}

Tabela 3

Freqüência do conhecimento de métodos contraceptivos e fonte principal de informação sobre esses métodos, das 179 pessoas do sexo masculino maiores de 17 anos. Cavunge, Ipecaetá, Bahia, Brasil.

\begin{tabular}{lrr}
\hline & $\mathbf{n}$ & $\%$ \\
\hline Conhece método contraceptivo & 174 & 97,2 \\
Sim & 5 & 2,8 \\
Não & & \\
& & \\
Fonte principal de informação & 6 & 3,4 \\
Família & 12 & 6,9 \\
Escola & 14 & 8,0 \\
Amigos & 24 & 13,8 \\
Meios de comunicação de massa* & 31 & 17,8 \\
Outros & 55 & 31,6 \\
Duas fontes diferentes & 32 & 18,4 \\
$\quad$ Três ou mais fontes & & \\
\hline
\end{tabular}

* Rádio e televisão. 
Métodos contraceptivos mais referidos, segundo a faixa etária, pelas pessoas do sexo masculino, maiores de 17 anos. Cavunge, Ipecaetá, Bahia, Brasil.

\begin{tabular}{|c|c|c|c|c|c|c|}
\hline \multirow[t]{2}{*}{ Método } & \multicolumn{6}{|c|}{ Faixa etária } \\
\hline & $\begin{array}{c}18-22 \\
(n=36) \\
n(\%)\end{array}$ & $\begin{array}{c}23-33 \\
(n=38) \\
n(\%)\end{array}$ & $\begin{array}{c}34-41 \\
(n=34) \\
n(\%)\end{array}$ & $\begin{array}{c}42-56 \\
(n=36) \\
n(\%)\end{array}$ & $\begin{array}{c}57-86 \\
(n=35) \\
n(\%)\end{array}$ & $\begin{array}{c}\text { Total } \\
(n=179) \\
n(\%)\end{array}$ \\
\hline "Camisinha" & $36(100,0)$ & $37(97,4)$ & $34(100,0)$ & $36(100,0)$ & $34(97,1)$ & $177(98,9)$ \\
\hline Pílula & $35(97,2)$ & $37(97,4)$ & $33(97,1)$ & $36(100,0)$ & $32(91,4)$ & $173(96,6)$ \\
\hline Laqueadura & $30(83,3)$ & $34(89,5)$ & $32(94,1)$ & $34(94,4)$ & $31(88,6)$ & $161(89,9)$ \\
\hline Coito interrompido & $31(86,1)$ & $35(92,1)$ & $32(94,1)$ & $30(83,3)$ & $23(65,7)$ & $151(84,4)$ \\
\hline Injetáveis & $26(72,2)$ & $35(92,1)$ & $29(85,3)$ & $32(88,9)$ & $26(74,3)$ & $148(82,7)$ \\
\hline Vasectomia & $20(55,6)$ & $28(73,7)$ & $32(94,1)$ & $33(91,7)$ & $27(77,1)$ & $140(78,2)$ \\
\hline Tabela & $16(44,4)$ & $23(60,5)$ & $22(64,7)$ & $25(69,4)$ & $9(25,7)$ & $95(53,1)$ \\
\hline DIU & $7(19,4)$ & $21(55,3)$ & $26(76,5)$ & $22(61,1)$ & $7(20,0)$ & $83(46,4)$ \\
\hline Creme vaginal & $6(16,7)$ & $15(39,5)$ & $18(52,9)$ & $20(55,6)$ & $5(14,3)$ & $64(35,8)$ \\
\hline Diafragma & $10(27,8)$ & $9(23,7)$ & $13(38,2)$ & $6(16,7)$ & $3(8,6)$ & $41(22,9)$ \\
\hline
\end{tabular}

Tabela 5

Conhecimento sobre os mecanismos de ação e prevalência de uso contínuo do método contraceptivo, pelo entrevistado ou companheira, de 179 pessoas do sexo masculino maiores de 17 anos. Cavunge, Ipecaetá, Bahia, Brasil.

\begin{tabular}{lcc}
\hline Método & $\begin{array}{c}\text { Mecanismo de ação } \\
n(\%)\end{array}$ & $\begin{array}{c}\text { Uso contínuo } \\
\mathrm{n}(\%)\end{array}$ \\
\hline Camisinha & $115(64,2)$ & $41(22,9)$ \\
Coito interrompido & $109(60,9)$ & $6(3,4)$ \\
Tabela & $7(3,9)$ & $4(2,2)$ \\
Pílula & $5(2,8)$ & $23(12,8)$ \\
Laqueadura & $2(1,1)$ & $38(21,2)$ \\
Vasectomia & $2(1,1)$ & $1(0,6)$ \\
Injetáveis & $1(0,6)$ & $3(1,7)$ \\
DIU & $1(0,6)$ & $1(0,6)$ \\
Diafragma & $1(0,6)$ & $0(0,0)$ \\
Creme vaginal & $0(0,0)$ & $1(0,6)$
\end{tabular}

não quiseram comentar sobre a freqüência de relações sexuais. Entre os 131 (73,2\%) entrevistados que possuíam relacionamento sexual fixo, oitenta e dois $(62,6 \%)$ disseram que conversam com as respectivas parceiras sobre métodos contraceptivos. Entre aqueles que responderam sobre a freqüência semanal da atividade sexual $(96,1 \% ; \mathrm{n}=172)$, esta teve média igual a $2,25( \pm 1,97)$, mediana de 2 , percentis $25^{\circ}$ e $75^{\circ}$ de 1 e 3 , respectivamente, e limites de zero a 9 vezes, sendo em $76,7 \%(n=132)$ até três vezes por semana. Não houve diferença estatistica- mente significante $(p>0,10)$ entre a freqüência semanal de relações sexuais dos, aproximadamente, $50,0 \%$ mais jovens ( $\leq 36$ anos) e a dos maiores de 36 anos de idade.

\section{Doenças sexualmente transmissíveis}

Quando perguntados sobre o que seria uma DST ou, as sinonímias, doença venérea e "doença do mundo", 166 (92,7\%) disseram que sabiam de que tais termos tratavam. Entre esses, dez $(6,0 \%)$ entrevistados referiram que a outra função da "camisinha" era a de prevenir DSTs; 12 $(7,2 \%)$ prevenir a AIDS, apenas; a maioria $(62,0 \%$; $\mathrm{n}=107$ ) referiu como função a prevenção tanto de DSTs, quanto da AIDS; somente um (0,6\%) referiu uma função incorreta e 40 (24,1\%) homens não sabiam se a "camisinha" tinha alguma outra função, além de "prevenir filhos”.

\section{Discussão}

A média de idade da população estudada foi alta (40 anos), o que pode ser explicado pelo fato de o povoado não oferecer perspectivas de inserção profissional para os mais jovens, os quais acabam migrando para cidades de maior porte em busca de melhores condições de vida. Também, as pessoas estudadas possuíam nível de escolaridade muito baixo. Como houve correlação positiva entre a idade e o tempo médio de residência em Cavunge, conseqüentemente as pessoas amostradas passaram grande parte 
da vida na localidade, onde não existem ações voltadas ao planejamento familiar. Estes fatos podem explicar o reduzido grau de informação apresentado pela população estudada.

Como observado por Chirinos et al. 12, para que o método contraceptivo escolhido seja efetivo é importante que se conheça de forma mais abrangente as características peculiares dos mesmos. Não obstante, apesar de grande parte dos entrevistados ter referido conhecer algum tipo de método, apenas os mecanismos de ação da "camisinha" e do coito interrompido são conhecidos pela maioria dos participantes, possivelmente porque esses são métodos que dependem de controle predominantemente masculino e portanto, o interesse dos homens pelos mesmos é maior, como observado na literatura 13. Sendo assim, as pessoas estudadas não tinham o conhecimento adequado que lhes possibilitem escolher, dentre os diversos métodos contraceptivos, o mais apropriado ao comportamento sexual individual, ou do casal.

Por sua vez, os métodos mais referidos são aqueles mais difundidos na sociedade e também mais divulgados pela mídia (camisinha, pílula e laqueadura), já os menos conhecidos são os dependentes da participação feminina ou menos difundidos na sociedade (diafragma, creme vaginal e DIU). Assim, há certa concentração do conhecimento em poucos métodos contraceptivos, o que, como também observado por Vieira 14 , pode ser prejudicial, na medida em que esta situação pode se refletir na prevalência do uso. Corroborando com esta afirmação, observou-se que métodos de esterilização (laqueadura e vasectomia) são bastante conhecidos - uso da laqueadura foi o segundo mais utilizado $(21,2 \%)$ - mesmo não sendo considerado por alguns autores como método contraceptivo por ser de difícil reversão 3 . Considerando ainda a esterilização, devemos enfatizar que este método traz implicações bioéticas importantes mas que fogem ao objetivo deste artigo.

Inversamente, um indício de ampliação das opções contraceptivas parece começar a existir, na medida em que, diferentemente do observado por Duarte 6, o conhecimento dos anticoncepcionais injetáveis foi elevado, o que pode se constituir em mais uma alternativa de contracepção, apesar destes serem também dependentes da participação feminina. Contudo, mesmo considerando ser a elevada freqüência do conhecimento sobre os anticoncepcionais injetáveis reflexo da maior divulgação, até por parte das indústrias interessadas, o casal tem o direito de receber informações sobre a possibilidade dos efeitos colaterais ou adversos dos mesmos, assim como de qualquer outro método; porque o grau de informação sobre o método anticoncepcional tem nexo com a aderência, ou não, ao uso contínuo do mesmo 15.

Apesar da maioria dos entrevistados possuir relacionamento fixo, pouco mais de um terço deles disse não estar utilizando nenhum método contraceptivo, o que evidencia a necessidade de atuação dos Programas de Planejamento Familiar na busca de oferecer aos casais informações necessárias para uma escolha consciente da quantidade de filhos e de quando tê-los, fato que, possivelmente, não ocorre na comunidade estudada, em vista da elevada média de filhos observada.

Quando perguntados sobre questões referentes às responsabilidades dos parceiros no que se refere ao cuidado dos filhos e a prevenção da gravidez, os entrevistados, aparentando um razoável senso de igualdade de gênero, referiram mais freqüentemente $(62,6 \%)$ que essas responsabilidades seriam do casal, seguida da responsabilidade exclusiva da mulher $(29,1 \%)$. Porém, incoerentemente, o responsável pela escolha do método usado foi o homem em quase metade das vezes $(45,6 \%)$, sendo o casal referido por apenas $11,4 \%$ dos casos. Isso mostra que os participantes sabiam da importância da participação conjunta do casal, o que é bom, e isto abre a perspectiva da maior aproximação da população masculina com as atividades referentes ao Planejamento Familiar. Mas, possivelmente devido às relações sociais existentes nesta comunidade onde o homem é visto como o chefe da família, ainda é este quem determina o método a ser utilizado pelo casal em quase metade dos casos, o que ajuda a fortalecer a hipótese de dependência e submissão feminina em comunidade semelhante a Cavunge.

Ficou evidenciado que a grande maioria dos entrevistados sabia o que era uma DST, e mais de dois terços conheciam a função de prevenção de alguma DST pelo método de barreira (“camisinha”), portanto, um fator que pode estar contribuindo para a escolha da "camisinha" como método contraceptivo.

Não existiram diferenças significantes entre o conhecimento e uso de métodos contraceptivos entre os que tinham e aqueles que não tinham um relacionamento fixo, contudo houve uma proporção maior de uso entre aqueles com idade inferior à mediana, o que possivelmente é reflexo de um maior nível de escolaridade e informação destes. Estes fatos demonstram a necessidade de que tais programas adotem ações baseadas, também, no nível prévio de informação dos usuários, e não apenas na existência ou não de relacionamento fixo entre os mesmos. 
Assim, ratifica-se a importância do conhecimento das características da população masculina local, a fim de direcionar melhor as ações dos Programas de Planejamento Familiar a serem implementados, obtendo-se resultados mais satisfatórios no que tange à promoção de um serviço de planejamento familiar adaptado às necessidades de determinada região.

\section{Resumo}

Os Programas de Planejamento Familiar vêm se preocupando predominantemente com a população feminina. Este estudo objetivou determinar o grau de conhecimento dos homens de uma comunidade rural do Estado da Bahia, Brasil,sobre os métodos contraceptivos. Dentre os 179 entrevistados, a preocupação com a prevenção de gravidez indesejada foi referida como responsabilidade do casal por $39,7 \%(n=71)$ e do homem por $26,8 \%(n=48)$. Os métodos mais conhecidos foram a "camisinha" (98,9\%) e a pílula $(96,6 \%)$, sendo os mais usados a "camisinha" (22,9\%), a laqueadura (21,2\%) e a pílula anticoncepcional (12,8\%). A maioria $(56,4 \%)$ referiu "sempre" utilizar contraceptivo. A opção pelo método é feita pelo homem em $45,6 \%$ dos casais. Estes resultados indicam a necessidade da promoção do maior número de métodos contraceptivos, possibilitando ao casal a melhor opção que se adeque ao comportamento sexual do mesmo. Também, há necessidade de priorizar a participação do casal nos Programas de Planejamento Familiar, na medida em que ainda são os homens quem predominantemente acabam determinando o método contraceptivo utilizado.

Anticoncepção; Comunidades Rurais;Gênero

\section{Colaboradores}

D. C. Espírito-Santo participou das etapas de revisão da literatura, de elaboração do projeto, de coleta e análise dos dados e de redação do artigo. J. TavaresNeto orientou a execução das etapas de revisão de literatura, de elaboração do projeto e de coleta de dados, e participou da análise dos dados e da redação do artigo. 


\section{Referências}

1. Brasil. Constituição da República Federativa do Brasil: texto constitucional de 5 de outubro de $1988 \mathrm{com}$ as alterações adotadas pelas Emendas Constitucionais de n. 1, de 1992, a 23, de 1999, e pelas Emendas Constitucionais de Revisão de n. 1 a 6, de 1994. Brasília: Congresso Nacional; 1999.

2. Corrêa S. Saúde reprodutiva, gênero e sexualidade: legitimação e novas interrogações. In: Giffin K, Costa SH, organizadores. Questões da saúde reprodutiva. Rio de Janeiro: Editora Fiocruz; 1999. p. 39-49.

3. Schor N, Ferreira AF, Machado VL, França AP, Pirotta KCM, Alvarenga AT, et al. Mulher e anticoncepção: conhecimento e uso de métodos anticoncepcionais. Cad Saúde Pública 2000; 16:377-84.

4. Arilha M. Homens, saúde reprodutiva e gênero: o desafio da ilusão. In: Giffin K, Costa SH, organizadores. Questões da saúde reprodutiva. Rio de Janeiro: Editora Fiocruz; 1999. p. 455-66.

5. Ferreira SL, Nascimento ER, Bessa LF, Ferreira TF. Opiniões de mulheres e homens a respeito da utilização dos métodos contraceptivos. Texto \& Contexto Enfermagem 1997; 6:246-59.

6. Duarte GA. Perspectiva masculina quanto a métodos contraceptivos. Cad Saúde Pública 1998; 14:125-30.

7. Carvalho MLO, Pirotta KCM, Schor N. Apoio: a forma predominante de participação masculina na regulação da fecundidade do casal. Saúde Soc 2000; 9:61-76.

8. Nolasco S. O mito da masculinidade. Rio de Janeiro: Editora Rocco; 1993.
9. Tavares Neto J, Barral A, Queiroz-Andrade M, Oliveira S. Caracterização da população do povoado de Cavunge, Bahia. Rev Baiana Saúde Pública 2003; 27:60-75.

10. Secretaria de Saúde do Estado da Bahia. Saúde reprodutiva: manual para prestação de serviços. Salvador: Secretaria de Saúde do Estado da Bahia, Governo da Bahia; 1998.

11. Brasil. Resolução no 196, do ano de 1996. Brasília: Conselho Nacional de Saúde; 1996.

12. Chirinos JL, Brindis C, Tye S, McCarter V. Differences and similarities in sexual and contraceptive knowledge, attitudes, and behavior among Latino male adolescent students in California, United States and Lima, Peru. Cad Saúde Pública 2001; 17:833-41.

13. Sociedade Civil Bem-Estar Familiar no Brasil. Pesquisa Nacional sobre Demografia e Saúde 1996. Rio de Janeiro: Sociedade Civil Bem-Estar Familiar no Brasil; 1997.

14. Vieira EM. Do women's attitudes towards abortion and contraceptive methods influence their option for sterilization? Cad Saúde Pública 1999; 15:739-47.

15. Canto de Cetina TE, Canto P, Luna MO. Effect of counseling to improve compliance in Mexican women receiving depot-medroxyprogesterone acetate. Contraception 2001; 63:143-6.

Recebido em 27/Jan/2003

Versão final reapresentada em 23/Set/2003

Aprovado em 23/Out/2003 